\title{
Domain Name
}

National Cancer Institute

\section{Source}

National Cancer Institute. Domain Name. NCI Thesaurus. Code C54076.

An internet address in alphabetic form. Domain names must have at least 2 parts: the part on the left which names the organization, and the part on the right which identifies the highest subdomain, such as the country (e.g., fr for France, uk for United King dom) or the type of organization (e.g., com for commercial, edu for educational). 